\title{
Los fondos antiguos de las bibliotecas universitarias: lugares desconocidos del patrimonio cultural mexicano ${ }^{1}$
}

\author{
María Idalia García Aguilar \\ Centro Universitario de Investigaciones Bibliotecológicas \\ de la UNAM , 04510, México D.F. Tel: 56230328 \\ E-Mail:pulga@cuib.unam.mx
}

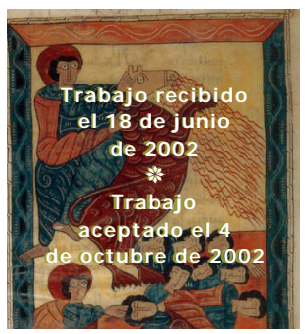

\begin{abstract}
RESUMEN
Los fondos antiguos de bibliotecas en México como parte del patrimonio cultural del país presentan características muy especiales que determinan sus condiciones de salvaguarda. O tros países han desarrollado un importante conocimiento sobre los materiales que estos fondos albergan, pero en México este conocimiento es parcial y limitado. Este trabajo analiza la problemática más general en materia de salvaguarda de los fondos antiguos conservados en México, resaltando el particular caso de las bibliotecas universitarias y valiéndose de un proyecto de investigación en desarrollo. Tratamos de enfatizar la necesaria colaboración e intercambio que debe existir entre las instituciones para transformar el futuro de los acervos patrimoniales. Finalmente se discuten algunos asuntos relevantes relacionados con uno de los principales objetivos de la salvaguarda: el registro y la identificación de los objetos culturales.
\end{abstract}

Palabras clave: Bibliotecas universitarias. Libros Antiguos. Fondo Antiguo. Patrimonio documental.

\section{RARE BOOKS SECTION IN UNIVERSITY LIBRARIES: UNKNOWN PLACES OF THE MEXICAN CULTURAL PATRIMONY}

\author{
MARÍA IDALIA GARCÍA-AgUILAR
}

\begin{abstract}
The section of Rare Books and Manuscripts as part of the Mexican cultural heritage has special characteristics which determine its conditions of safeguard. O ther countries have developed many and important knowledge on the materials existing in these sections, but in Mexico this is incomplete and limited. The general problem of safeguarding documentary heritage is here described by means of a research in progress, with particular emphasis in the university libraries. We pretend to underline the collaboration and exchange
\end{abstract}

1 Este trabajo forma parte del Proyecto de Investigación Conoernuestramemmia dbametal: loskienes bibliogáficos nunivesidades mexicanas quesefinamia con e apoyodeCONACYT (J30799-H). 
which is necessary to have with the official institutions in order to be able to transform the future of these collections. Discussion is made of some relevant matters related with one of the main aims of the safeguard: the registration and identification of cultural objects.

Keywords: University Libraries. Antiquarian Materials. Rare Books Section Library. Documental Heritage.

\section{“Tobpensamientodeniva deunasensaaión contraniada"}

Emil Ciaran

\section{INTRODUCCIÓN}

—n toda la riqueza cultural de un país existen siempre unos objetos más valorados que otros. Las razones que justifican esta distinción están determinadas por múltiples factores históricos, sociales, culturales, económicos e incluso políticos. Al analizar estos factores se puede descubrir el recorrido que hace una sociedad determinada al valorar sus objetos culturales, lo cual sin duda define el lugar queesos mismos objetos ocupan en el panorama cultural de ese país. Estelugar define sobre todo la posición que ocupan los objetos patrimoniales dentro de las políticas culturales de un estado.

México es un país que se distingue por una enorme riqueza cultural que ha heredado de generaciones anteriores, y también por una historia compleja que precisamente por esta característica ha producido una diversidad cultural que se manifiesta no sólo en las prácticas culturales de los distintos grupos sociales, sino también en la producción de numerosos objetos que hoy valoramos de forma especial. Empero, existe una gran diferencia entre el discurso que avala la existencia y conservación de bienes culturales, y las condiciones reales que garantizan su adecuada salvaguarda.

Esta dicotomía está presente en nuestro país sobre todo para aquellos objetos que se conservan en las bibliotecas y los archivos, ya sean públicos o privados. En lo que se refiere particularmente al patrimonio custodiado por muchas bibliotecas mexicanas, se observa un reconocimiento discursivo del libro como objeto cultural y vehículo de transmisión de ideas que sin embargo no ha logrado transformar sensiblemente la realidad institucional de las bibliotecas mexicanas. Todas estas ideas manifestadas en múltiples espacios políticos, académicos o culturales, deberían convertirse progresivamente en acciones concretas capaces de cambiar las condiciones de salvaguarda de los objetos culturales conservados en bibliotecas, pero esto no ocurre.

La conservación de los bienes culturales de distintanaturaleza, así como su transmisión, dependen en gran medida del lugar que ocupe esta importante tarea en la 
política cultural de un estado. No obstante, para que la conservación de bienes culturales sea prioridad para las responsabilidades de los Estados, los objetos culturales deben gozar de un reconocimiento social, condición sinequanonpara garantizar no sólo la conservación sino la transmisión de los objetos valorados culturalmente.

En efecto, las tareas de conservación no podrían emprenderse sin el conocimiento pleno de los objetos culturales que se quieren conservar. Por esta razón, el primer paso necesario de toda acción de conservación es el registro e inventario de los bienes implicados. O bviamente si no se conoce el universo de bienes culturales es imposible diseñar programas eficaces de conservación para ellos. De hecho este mismo conocimiento determinará las acciones de difusión, acceso y disfrute del bien cultural. Acciones que propiciarán, en gran medida, el aprecio y reconocimiento social de ese patrimonio cultural. Sin duda esta misma valoración social tiene también un papel coadyuvante en la transmisión de los bienes culturales a las generaciones venideras.

\section{ENTRE LAS MÚ LTIPLES CARAS DEL PATRIMONIO CULTURAL MEXICANO: EL LUGAR DE LIBROSY DOCUMENTOS}

México, al igual que otros países, se precia de tener un rico legado cultural de las generaciones pasadas. Este vasto patrimonio está presente en prácticamente todas las formas de representación que el país hace de sí mismo. A demás, este patrimonio también se ha convertido en una fuente de recursos importante, aunque no necesariamente en un factor de desarrollo sostenible. En efecto, el patrimonio cultural representa una riqueza que puede ser explotada, pero queantes debe ser conservada para cumplir con esa finalidad. La conservación de este patrimonio adquiere entonces dos matices importantes: aquél que es necesario para hacer un aprovechamiento económico y, el más importante, el que reconoce esa riqueza como una herencia social que adquiere su importancia precisamente por la transmisión que hace en el tiempo.

Por estas razones las cuestiones patrimoniales han cobrado mayor importancia en las preocupaciones de los estados. De esta forma, la manera tradicional de entender el patrimonio como sólo un inapreciable legado cultural, ha evolucionado hasta ser reconocido como unaimportantefuente de recursos económicos y de desarrollo para las personas que lo poseen. ${ }^{2}$

Ciertamente, uno de los mayores problemas que ha estado presente en la conservación del patrimonio cultural es el enorme gasto económico necesario para hacer las tareas de salvaguarda. ${ }^{3}$ En algunos casos esto es más necesario que en otros, pero con el aumento de los bienes patrimoniales la inversión necesaria para

2 Cfr. Rosa Campillo Garrigos. Lagestiónyd gestardd patrimmioailtural. Murcia: Editorial KR, 1998. p. 40

3 Entre estas tareas se incluye desde la conservación preventiva o la legislación necesaria de protección, hasta los productos culturales y las tareas de difusión. 
garantizar la salvaguarda crece exponencialmente. Por esta razón el aprovechamiento económico también representa hoy una opción invaluable para garantizar y ayudar a la salvaguarda.

También habría que considerar la existencia de una enorme diferencia entre los textos y los discursos que se elaboran sobre bienes culturales, y la realidad subsistente en cuanto al verdadero aprovechamiento de los mismos. Es en esta perspectiva desde la que debemos analizar la situación actual de los recursos culturales conservados en bibliotecas y archivos mexicanos, especialmente aquellos que se custodian en instituciones de educación superior, tanto por su número como por su representatividad.

Analizar las problemáticas que se relacionan con este patrimonio implica considerar aspectos tan diversos como leyes y normas; características institucionales; recursos económicos, materiales y humanos, y también las peculiaridades de los objetos conservados que nos permiten hacer la distinción entre unos y otros.

Resulta prácticamente imposible pensar que bastan unas líneas para plantear y ana lizar distintas problemáticas de igual importancia, y aportar así soluciones idóneas para salvaguardar una riqueza de dimensiones tan enormes. En lo que se refiere al patrimonio conservado en las bibliotecas mexicanas, dicho análisis resulta aún más complicado debido a la ausencia casi total de discusión sobre la materia. D ebemos por tanto comenzar a plantear preguntas por lo que se refiere a los fondos antiguos en general y sobre aquellos conservados en bibliotecas universitarias en particular.

Si bien México cuenta con instituciones específicas así como programas y políticas definidos para salvaguardar el patrimonio cultural nacional, y también una legislación específica destinada a esta materia, podemos afirmar que no existe un espacio institucional en el que se analicen y propongan soluciones idóneas para salvaguardar esta enorme riqueza custodiada en archivos y bibliotecas. Una riqueza que, como cualquier acervo de esta naturaleza, está en permanente crecimiento. No hay que olvidar que el patrimonio que caracteriza a estas instituciones es, tanto lo que hemos heredado, como lo que construimos para heredar. En suma, representa lo que existe actualmente y lo que existirá en el futuro.

En efecto, el panorama cultural mexicano en cuanto a este legado cultural se refiere, deja mucho que desear. A pesar de que estos recursos están considerados como parte de ese patrimonio cultural desde diversas perspectivas y de que cuentan con la protección legal vigente, no hemos logrado consolidar el valor patrimonial de estos objetos a partir de su propia producción como parte fundamental de una política cultural definida. Ciertamente, en el futuro, la producción bibliográfica actual pasará a formar parte de esa herencia patrimonial, por lo que debería irse integrando de forma gradual al conjunto ya existente. Sin embargo los ordenamientos jurídicos que aplican, no han logrado relacionar unos aspectos con otros para que este patrimonio se vea enriquecido con nuestro propio desarrollo cultural. Se crea así una difícil situación que con el tiempo solamente se recrudece.

D urante el siglo XX y lo que va del XXI desdela Constitución de 1917, se han elaborado en México cuatro leyes específicas orientadas a regular la protección de los 
bienes culturales del país. En todas estas leyes (de 1930, 1934, 1970 y 1972), han estado presentes prácticamente sin ninguna variación, el libro y el documento como objetos culturales. Estos objetos son considerados en nuestra tradición jurídica como bienes públicos y muebles, cuya naturaleza no es normalmente sustituible. Es decir, están considerados como invaluables.

En esta consideración y reconocimiento observables no sólo en las leyes federales sino también en las estatales, podemos encontrar dos características que cabe distinguiry que afectan al patrimonio conservado en las bibliotecas mexicanas, independientemente de lainstitución o el dominio al que pertenecen. La primera de estas características es el lugar que se reconoce para los objetos patrimoniales conservados en bibliotecas, tales como manuscritos, incunables, libros antiguos, ediciones del siglo XIX y otros. Estos objetos están reconocidos en la LeyFederal sobreZnasy Monumentos Arquedógicos Históricos, Artísticos de 1972, como "monumentos históricos", y se consideran como objetos dignos de conservar. Sin embargo, esta misma deferenciano se establece para la biblioteca como institución de custodia. Estaapreciación se desprende de la lectura del texto legal, porque aun cuando la ley establece la misma condición para los objetos que para las colecciones de ellos, no se define claramentela función institucional de la biblioteca como guardián de la memoria bibliográfica. La segunda característica es la ausencia de reconocimiento o de definición de los objetos custodiados por las universidades, cualquiera que sea la naturaleza de aquellos.

El análisis sobre la evolución de los objetos considerados en las leyes de protección del patrimonio cultural de México, nos muestra, sin lugar a dudas, el lugar que tienen todos los objetos que nuestro país reconoce como culturales, y por tanto, el lugar que adquieren como parte de esa herencia del patrimonio cultural. Por ejemplo, el exclusivo reconocimiento al patrimonio arqueológico como patrimonio nacional en la citada ley de 1972. ${ }^{4}$

Pero si consideramos las leyes del Estado mexicano en materia de bibliotecas, 5 observaremos que la condición patrimonial del libro está permanentemente ausente del ordenamiento jurídico. ¿Por qué sucede esto? Es muy probable que la respuesta esté determinada por el valor social que tanto el libro como la biblioteca tienen actualmente en México. Pero, a diferencia de otros países, el nuestro no cuenta aún con un soporte estadístico importante del medio cultural como para definir con claridad este valor hipotético al que nos referimos.

Ciertamente no sólo se trata de números sino de la forma en que la sociedad mexicana considera a la biblioteca en el marco de sus preocupaciones sociales. En este sentido, recientemente se ha mencionado la falta de lectores como pretexto para no establecer un programanacional dedicado a resolver el problema de la lectura. Empero, lo

$4 \quad$ Así se establece en el artículo 270 de la ley.

5 LaLeyGeneal deBiblicterasfue publicada en 1988, y está dedicada a definir la estructura y funcionamiento del Sistema Nacional de Bibliotecas Públicas. 
que no se ha definido claramente es si la existencia de bibliotecas garantiza necesariamente la existencia de lectores. La historia de México puede demostrar que ésta no es una ecuación perfecta. Nuestro país siempre ha tenido bibliotecas ricas e importantes, pero esto no ha propiciado necesariamente un aumento de lectores.

D e ahí que sea importante cuestionar la apreciación social que tenemos los mexicanos de las bibliotecas, especialmente si pretendemos inculcar y transmitir una conciencia de salvaguarda de los objetos conservados en bibliotecas, sean éstos antiguos o contemporáneos. Si partimos del presupuesto de que las leyes se elaboran para resolver problemas o preocupaciones presentes en la sociedad, tendremos que aceptar que el valor patrimonial del libro y de la biblioteca no están claramente definidos ni por, ni para la sociedad mexicana. Este valor, es sólo un hilo de la inmensa madeja que representala salvaguarda de los bienes culturales que se conservan en las bibliotecas mexicanas. Es decir, ¿cómo definir el reconocimiento legal deunos objetos custodiados en una institución tan particularmente reconocida como lo es la biblioteca? Presuponer que el reconocimiento de una colección de ciertos objetos, puede entenderse al mismo tiempo, como la afirmación de que los mismos están dentro de un espacio institucional, es construiruna conjetura que no ayuda mucho.

En efecto, si la biblioteca e incluso el archivo son considerados establecimientos culturales de importancia y también reconocemos que sus colecciones patrimoniales son de gran valor, ¿entonces por qué no se los ha considerado en el marco legal y general de protección del patrimonio cultural mexicano? Es decir, si reconocemos que un conjunto de objetos como libros y documentos, o una colección de ellos, son insustituibles o merecen ser conservados en el país ¿por qué no se ha establecido en las leyes patrimoniales el lugar y reconocimiento de esas instituciones en donde por norma general aquellos se custodian? Ciertamente es difícil darle una respuesta precisa a estas preguntas. Especialmente si se considera que la tendencia internacional en materia legal sobre patrimonio cultural, ha favorecido el reconocimiento de la protección legal de la biblioteca y del archivo en su función patrimonial. Así, al mismo tiempo se garantiza la salvaguarda de los objetos culturales que se custodian en estos establecimientos y, desde la misma perspectiva, a los establecimientos.

Pero en nuestro país, el panorama de la salvaguarda de bienes patrimoniales, bibliográficos o documentales, está desmembrado y no resulta eficaz para salvaguardar esos objetos. El reconocimiento por parte de una ley, sólo permite definir el campo de acción y las formas de actuar para salvaguardar, pero la ley por sí misma no podría resolver los problemas de conservación de los elementos patrimoniales. Existe un punto crucial e inevitable que orienta y define todas las tareas de la salvaguarda: el problema del registro de los bienes culturales.

El registro representa el punto de partida nodal, debido a que en función del conocimiento del número de objetos, las características que los distinguen, así como los lugares en que se encuentran, puede elaborarse un programa de conservación más acorde con la realidad y, por tanto, más ajustado a la situación de los objetos valorados culturalmente. En este sentido y a pesar de su importancia, los bienes de naturaleza 
cultural que se custodian en las bibliotecas mexicanas no han sido registrados cabalmente. Hoy, y a pesar de muchos esfuerzos realizados en ese sentido, no se cuenta con ningún catálogo colectivo de los bienes conservados en nuestras bibliotecas. Por tanto no podemos saber cuántos, cuáles y de qué tipo son, cuál es su estado de conservación y menos aún cuáles son las características que los hacen especiales 0 los distinguen a unos de los otros.

La existencia legal de dos registros nacionales y públicos, uno para los bienes arqueológicos e históricos, y otro para los bienes artísticos, no resuelve en absoluto el problema del registro en las bibliotecas mexicanas, aun cuando a ambos se los considere legalmente como monumentos históricos. Estos registros nacionales tampoco resuelven el problema de los archivos, cuya complejidad es una tarea de muy largo plazo. Recordemos que la mayor parte de los archivos suele medir sus dimensiones culturales en kilómetros de documentación.

Cabe pensar que el problema del registro de estos particulares bienes - bibliográficos y documentales-, debió empezar por depositar la responsabilidad en las mismas instituciones que los conservan. ¿Q uién mejor para elaborar un modelo de registro adecuado a su naturaleza? Pero ninguna de las leyes mexicanas en esta materia elaboradas durante el siglo XX, consideraron o definieron así el problema. Contrariamente, la misma tarea se deposito también en otras instituciones del Estado mexicano. Habría que preguntarse ¿por qué han estado fuera de esta perspectiva dichas instituciones?

O tro punto a considerar es la dependencia institucional de la Biblioteca Nacional de México y del Archivo G eneral de la Nación (AGN), la primera bajo la estructura institucional de la Universidad Nacional Autónoma de México (UNAM), y el segundo bajo la dependencia de la Secretaría de Gobernación. ¿No resulta extraño que dos instituciones culturales de tal importancia estén fuera del ámbito del órgano coordinador de la cultura en México? A partir de 1990, esta coordinación se depositó en el Consejo Nacional para la Cultura y las Artes (CONACULTA), órgano del que dependen, entre otras instituciones culturales, el Instituto Nacional de Antropología e Historia (INAH) y el Instituto Nacional de Bellas Artes (INBA). Estas últimas por ley vigente, deben realizar el registro de la riqueza cultural de México.

Esta característica institucional supone dificultades pero no imposibilidades. Al final todas son instituciones públicas y, como tales, parte del Estado. Existe otra consideración en las leyes mexicanas de esta materia: la fórmula jurídica que deposita en una institución la idea de "patrimonio propio". Sin duda toda institución requiere de recursos apropiados para funcionar, pero éstos deben ser separados claramente de lo que es la propiedad nacional; especialmente en lo que se refiere a los bienes culturales custodiados en cualquier dependencia, sea ésta pública o privada. E ste es el caso particular de las universidades, creadas bajo la citada fórmulajurídica de "patrimonio propio", noción que incluye numerosos bienes de naturaleza cultural de importancia nacional. 
Sin duda, todo registro de objetos culturales debe realizarse bajo una coordinación nacional, lo cual debe estar básicamente a cargo y ser responsable del diseño de modelos de registro para cada uno de los objetos del patrimonio cultural. También debería concertar las tareas de registro e inventario de las instituciones custodias, así como almacenar y sistematizar la información compilada, con la finalidad de contar con mejores instrumentos que permitan diseñar una política nacional de salvaguarda del patrimonio cultural mexicano.

Justamente por esta razón, la responsabilidad de coordinación del registro para los bienes bibliográficos, debió depositarse, por ley, en la Biblioteca Nacional, aun cuando se encuentre bajo la tutela de la UNAM. Y la misma responsabilidad legal e institucional sobre los bienes documentales debió depositarse en el Archivo G eneral de la Nación. Responsabilidad institucional que debería reflejarse en los ordenamientos jurídicos correspondientes. Actualmente ninguna de estas instituciones cuenta con una ley propia que se relacione con otras queabordan la misma materia.

Nuestro país ha sido ingenuo al pretender que el registro y la conservación de todos los bienes culturales denuestro inmenso patrimonio puede hacerse eficazmente con sólo la labor de dos institutos nacionales; aun cuando existan centros regionales como los del Instituto Nacional de antropología e Historia (INAH). Registrar bienes culturales es una actividad de orden e importancia nacional, pero también una tarea que debe realizarse en coordinación, colaboración eintercambio. Entre más instituciones participen, más rápidamente podrá conocerse la dimensión del patrimonio cultural mexicano y, por supuesto, los problemas de conservación, protección, restauración, y custodia que éste enfrenta.

"En realidad, la cantidad de patrimonio cultural es demasiado vasta para ser abarcada por el INBA y el INAH, razón por la cual éstas no son las únicas instituciones que actualmente efectúan trabajos de... [conservación]. Existen otras instituciones que... [actúan] por su cuenta [sobre] los bienes que tienen a su cargo, como por ejemplo la D irección G eneral del Patrimonio Universitario de laUNAM, el Archivo General de la Nación (dependiente de la Secretaría de G obernación), o el Museo Franz Mayer (privado)" ${ }^{6}$ Sin embargo todas estas acciones no siguen un programa nacional y por tanto representan acciones aisladas.

Por otra parte, habría que precisar que los libros del pasado son completamente diferentes a los modernos; su estructura, composición, tratamiento y adecuada conservación, requieren necesariamente de formación especializada. Lamentablemente en nuestro país prevalece la opinión de que dicha formación es inútil y tiene poca relación con el desarrollo de la investigación científica, que no es lo que sucede en España, Francia, Italia e incluso E stados Unidos, donde esta formación especializada tieneunaalta estimano sólo en las escuelas profesionales sino también en los grupos de investigación o en las asociaciones profesionales. En México, la preocupación de

6 Rebeca Alcántara Hewwit. Un análisis cútico dela teena dela restauracón deCesareBrand. México: INAH, 2000. p. 95 
los profesionistas por el legado bibliográfico parece ocasional y por ello el tema no es importante en el desarrollo del conocimiento.

Esta carencia nos coloca en un lugar poco recomendable y revela una práctica constante de descuido. A la fecha, son pocas las instituciones que se han preocupado por ofrecer espacios para esta formación tan particular y necesaria para preservar una riqueza en permanente riesgo. Sin embargo, existen numerosos proyectos de investigación financiados por diversas instituciones cuya finalidad es registrar los importantes testimonios que guardan los objetos documentales del pasado. Si no se tiene conocimiento especializado, resulta complejo establecer condiciones y particularidades sobre un tipo de bien cultural y no se vincula a éste con el conjunto general del que se desprende esta valoración; es decir, las bibliotecas que los han custodiado durante generaciones.

Con todo, los proyectos pasados y presentes no han logrado estructurar un espacio nacional que permita delinear un futuro diferente para los fondos antiguos conservados en México. Conocer el conjunto general de los materiales del fondo antiguo de las bibliotecas, permitiría establecer cuáles de ellos son los materiales más relevantes o singulares, e incluso únicos, y determinar una protección especial. Por otra parte, esos materiales por razones de conservación no pueden gozar de un acceso público ilimitado. Todo lo contrario, los espacios de custodia son lugares de acceso restringido, por lo que deben aplicarse mecanismos y formas idóneas que permitan conocer y disfrutar estos materiales como bienes del patrimonio cultural de todos los mexicanos.

Sin embargo, habría también que pensar que los ricos fondos de bibliotecas mexicanas no sólo están integrados por libros antiguos. Ciertamente existen en estos espacios otro tipo de materiales que por diversas razones históricas han pasado a formar parte de estos acervos. El fondo antiguo representa un complejo mundo de objetos que debe ser analizado cuidadosamente con objeto de definir claramente qué se entiende por este concepto. Efectivamente, la composición de este particular fondo se ha distinguido siempre por los libros y los documentos ahí conservados.

Por el contrario, cada uno de los materiales que integran este fondo presenta características genéricas que subdividen al acervo en conjuntos especiales de objetos, y la adecuada salvaguarda de éstos requiere un conocimiento especial. Así, podemos identificar entre estos grupos distintos a los incunables, los manuscritos, los libros antiguosy los libros del siglo XIX, pero también a otro tipo de materiales como fotografías, mapas, documentos históricos, ediciones especiales, facsimilares, microfilms, diapositivas, libros impresos durante el siglo XX eincluso ediciones electrónicas. Todos estos objetos por diversas razones constituyen parte del fondo antiguo.

D esde la perspectiva del programa Memoria del Mundo de la UNESCO, el problema de conocimiento del fondo antiguo se complica. Esto es así porque dicho programa define al patrimonio documental como los libros, manuscritos y documentos valiosos conservados en bibliotecas y archivos, así como aquellos documentos 
contenidos en cualquier medio o soporte tales como documentos audiovisuales, reproducciones digitales y a las tradiciones orales. ${ }^{7}$ Es decir, la mayor parte del material que integra al fondo antiguo puede ser considerado como parte del patrimonio documental mexicano.

A pesar de esta relación directa establecida entre el fondo antiguo y el patrimonio documental, y de la creación del citado programa internacional citado en 1992, esta perspectiva patrimonial no se ha integrado a nuestras leyes y normas vigentes. Por otra parte, tampoco hemos definido claramente qué entendemos por fondo antiguo, lo que luego nos permitiría precisar qué tipo de objetos lo integran. Hemos intentado, en colaboración, abordar este problema conceptual en un trabajo reciente. ${ }^{8}$ Pero aun cuando existiera una definición justificada se requeriría del consenso y de la aplicación del concepto para fortalecerlo, y sólo después de esto se podría integrarlo a los textos jurídicos.

Éste es, en mi opinión, el mayor problema que existe para analizar la problemática del fondo antiguo de las bibliotecas como un aspecto del patrimonio cultural; y se debe principalmente a que los profesionistas y quienes se encargan de estos fondos, comprenden y definen de manera diferente los objetos que forman parte de este fondo antiguo. Por ejemplo, en el caso particular de México, el concepto "incunable", que se refiere a los impresos producidos desde la invención de la imprenta de tipos móviles hasta 1500, ${ }^{9}$ pero que también se aplica a los impresos producidos en la Nueva España durante el siglo XVI. ${ }^{10}$

En efecto, la primeraimprenta americana se estableció en territorio novohispano en 1539, por lo que se ha dado por denominar como "incunables americanos" a los libros producidos desde esa fecha hasta el año de 1600. Sobre este concepto y su definición, como sobre otros conceptos relacionados con el fondo antiguo, no se ha establecido ninguna precisión derivada del conocimiento especializado. D e haberlo hecho, habríamos posibilitado formas de comprensión que deberíamos transmitirle a las nuevas generaciones a través de la formación profesional.

Así, esta diversidad conceptual no permite definir claramente el alcance del problema del fondo antiguo de México. De tal forma, cuando se cuestiona el tipo de materiales conservados en un fondo particular, podemos suponer que la información proporcionada padecerá de esta diversidad, y necesariamente se requerirá de

7 Abdelaziz Abid. Memwiada Munda consevandonuestropatimmiodbamettal. México: Comisión Nacional de los Estados Unidos Mexicanos para la UNESCO : UNAM: UAEM: UAEH, 1998. p. 10

8 Crr. Idalia G arcía y Miguel Ángel Rendón. "El fondo antiguo: su estructura conceptual", en Bina na: reista decommicadón, altura ytemdớa Vol. 1 (diciembre 2001). D isponible en:

http:/ / www.uem.es/ binaria/ [Consultado: junio 2002]

9 Konrad Haebler. Introduciónal etudiodelosinamables Madrid: Ollero \& Ramos, 1995. p. 18

10 Por ejemplo la autora escribe que se entiende por incunables los impresos producidos hasta el año de 1550. Cfr. Maria Marsá Vila. El fondbantiguoenlabiblideea Gijón: TREA, 1999. p. 113 
una confirmación directa con el material para saber si en efecto los materiales reportados como incunables efectivamente lo son. ${ }^{11}$

Sin duda, también contribuye a mantener esta situación el hecho de que este tema no exista en la formación profesional y en la investigación especializada del país. Son muchos los aspectos por considerar y en los que la claridad de los conceptos resulta fundamental. En este sentido no podemos obviar el desarrollo del conocimiento realizado en otras latitudes y utilizar los conceptos como corresponde. En esta situación, definir con claridad el lugar que ocupael patrimonio documental dentro del panorama del patrimonio cultural mexicano resulta toda una aventura, pues hay que diferenciar entre lo que se reconoce como un objeto, lo que se establece sobre ésteen términos de protección legal, y lo que se conoce deese objeto en relación con su tiempo histórico y su relación con aquellos otros objetos de las mismas características.

Lo que sí podemos afirmar es que la poca relación existente entre todos aquellos aspectos que necesariamente tienen que ver con la salvaguarda de un objeto del patrimonio documental, está directamente relacionada con dos cuestiones: el escaso conocimiento que tenemos en México de estos objetos y, por tanto, el valor social que adquieren éstos en relación con otros objetos patrimoniales.

Podríamos suponer que el aumento de conocimiento especializado podría ser transmitido a la sociedad, para que ésta construyera el valor cultural de estos objetos mediante sus propios procesos históricos. Este proceso podría ser interpretado así, si recordamos que los fondos antiguos por razones de conservación son de acceso restringido, y que además su adecuada comprensión exige conocimientos previos. Sin la investigación especializada el público general no podría determinar el número de objetos conservados ni tampoco el valor cultural y social que los distingue. Por lo tanto, la sociedad tampoco podría demandarleal Estado y sus instituciones una protección eficaz que garantizara la permanencia del legado cultural que representan los fondos antiguos.

Un legado de esta naturaleza requiere de acciones concretas planificadas a largo plazo y de una finalidad definida: conseguir que la sociedad mexicana conozca su existencia y particularidad, que aprecie su valor cultural y las razones que lo fundamentan; para garantizar así la adecuada salvaguarday transmisión, y asegurar que las generaciones venideras tengan la misma posibilidad de recrear su pasado que nosotros tenemos hoy.

11 Hemos enfrentado esta situación desde 1999, cuando desarrollamos el proyecto de investigación universitario "El patrimonio D ocumental en México". En varias ocasiones, las bibliotecas reportan incunables que en efecto no corresponden con las características que los especialistas han de terminado. Este mismo caso se aplica a otros materiales reportados y datados en un periodo específico que al revisarlos no resultan ser lo que se afirmaba. 


\section{LA UNIVERSIDAD COMO LUGAR DE CUST ODIA DE BIENES CULTURALES}

La función de las universidades, tanto públicas como privadas, en cuanto a la conservación y difusión de la cultura, es incuestionable. D icha condición está básicamente determinada porque uno de los pilares que las caracterizan como instituciones en su labor educativa y cultural es, precisamente, la conservación y difusión de los valores culturales.

Las universidades representan una vieja y rica herencia medieval que se ha diseminado por todo el mundo. Con historias diferentes, las universidades del mundo han ido conformando un acervo patrimonial de enorme riqueza y diversidad que por sus dimensiones y riqueza se ha convertido en una responsabilidad tanto de la universidad como de la nación ala que pertenece. En esteconjunto patrimonial, vinculado directa 0 indirectamente con la historia universitaria, existen numerosos objetos culturales de distinta naturaleza tales como bienes inmuebles, artísticos, históricos, científicos, documentales y, por supuesto, bibliográficos.

Si bien no es una característica genérica que todas las universidades posean un importante conjunto de bienes bibliográficos como parte de su patrimonio, sí es característico que aquellas que se precian de tener una larga historia y tradición tengan bajo su custodia testimonios materiales de la evolución del conocimiento y del saber de la sociedad que las ha creado, sostenido y apuntalado como instituciones culturales.

La biblioteca universitaria, tal y como la conocemos, no nace al mismo tiempo quelainstitución. En efecto, "las Universidades no fueron madrugadoras en lavaloración del libro y labiblioteca como instrumento imprescindibles para el desarrollo de sus actividades". ${ }^{12}$ Este vínculo tan estrecho se dará con posterioridad. Particularmente, durante el siglo XVIII, la universidad y su biblioteca establecerán un lazo que las convierte en la actualidad en partes indisociables de la misión que tienen las instituciones de educación superior.

Es así, paralelamente al desarrollo del conocimiento que impulsó la Ilustración, como las bibliotecas universitarias se convirtieron progresivamente en ejes importantes de la institución. D urante este proceso de fortalecimiento, sus acervos bibliográficos se enriquecieron por diferentes procedencias institucionales y personales, que en ocasiones le dieron renombre ala propia institución universitaria. Esta riqueza cultural de las bibliotecas universitarias creció notablemente durante los siglos XIX yXX mediante los diferentes procesos políticos y sociales que han ido definiendo a las instituciones de educación superior.

En México surgieron los establecimientos universitarios con la introducción de la cultura occidental durante la colonización española del siglo XVI. Aun cuando los

12 D ario Villanueva Prieto. "Presentación". En Ex libis Univesitatis e patrimmiodelasbiblidtecas universitariasespañdas Madrid: Conferenciade Rectores delas Universidades E spañolas, 2000.p. [13] 
historiadores ${ }^{13}$ se han ocupado mucho por dilucidar cuál de las tres instituciones universitarias - México, Lima o Santo D omingo-, puede ser considerada como la primerainstitución universitaria de América, la Real y Pontificia Universidad deMéxico constituye un rico pasado histórico para la educación superior del país. A pesar de su noble origen, esta institución "no contó con su biblioteca hasta los últimos años de la vida colonial" . ${ }^{14}$ Sin embargo, el periodo novohispano fue rico en bibliotecas conventuales, de colegios y seminarios, y de particulares. De este pasado dan cuenta numerosos bienes bibliográficos conservados y repartidos a lo largo del territorio nacional.

Al igual que en Europa todas estas bibliotecas también enriquecieron sus fondos bibliográficos con la expulsión dela Compañía de Jesús. Y del mismo modo el movimiento ilustrado en México fortaleció y enriqueció numerosas bibliotecas de la época, como la Real Academia de San Carlos o la del Real Colegio Seminario de Minería. Posteriormente se enriquecieron en el siglo XIX con la aplicación de las Leyes de Reforma de 1859, que nacionalizaron los bienes eclesiásticos. D espués de 1810, tras el establecimiento del Estado decimonónico y laformación delosinstitutos tecnológicosy científicos de los estados, se garantizaría la transmisión de importantes bienes culturales a las universidades mexicanas, entre los que no hay que olvidar a los fondos antiguos.

Actualmente muchas universidades mexicanas, tanto públicas como privadas, se precian de poseer un rico legado cultural manifestado en diversos objetos. Un legado que se ha enriquecido notablemente con el tiempo y la historia particular de cada una de estas instituciones. Por sus particularidades, las universidades también reciben donaciones y comodatos que contribuyen a enriquecer sus acervos culturales.

Toda esta riqueza participa y está presente en la vida cotidiana de las universidades a través de sus actividades culturales; sin embargo, al igual que ocurre con el panorama cultural del país, no todos los objetos patrimoniales gozan de los mismos privilegios. É ste es el caso de las bibliotecas universitarias que custodian fondos antiguos y que no cuentan con la misma representación e importancia en la vida cultural de las universidades.

Actualmente las bibliotecas universitarias ocupan un importante lugar en el desarrollo de la investigación especializada. Sus fondos bibliográficos, en constante crecimiento, pueden ser considerados fundamentales para el desarrollo del conocimiento porque representan los principales depósitos de colecciones científicas. En el mundo, muchas de estas bibliotecas albergan un pasado rico y complejo que se refleja en colecciones patrimoniales de gran valor, las cuales en los últimos tiempos se están convirtiendo en una preocupación importante de las instituciones que las tutelan. Ciertamente la evolución de cada biblioteca universitaria es distinta y por tanto su

13 Margarita Menegus Bornemann y Armando Pavón Romero. "La Real Universidad de México. Pa norama historiográfico”, en HistonadelaUnivesidadCdonial: avancesdeinvestigacón México: UNAM. Centro de Estudios sobre la Universidad, 1987. p. 73

14 Ignacio O sorio Romero. Lasbibliderasnoudispanas México: SEP. D irección G eneral de Bibliotecas, 1986. p. 209 
riqueza bibliográfica varía considerablemente. Pero desde la década de los ochenta del siglo XX, estas colecciones culturalmente valiosas están cobrando una mayor importancia y por ello una mayor visibilidad en el medio cultural de todos los países y por supuesto en el panorama universitario.

Sin embargo la aplicación de esta tendencia se ha aletargado en nuestro país. Por ello resulta preocupante observar que la mayor parte de las universidades mexicanas que cuentan con un fondo antiguo no estén participando activamente en los espacios que se han creado para trabajar sobre esta riqueza o en su defecto en el establecimiento de espacios propios. La universidad representa un espacio institucional privilegiado para el rescate y difusión de los fondos antiguos precisamente porque integra en un solo sitio la formación profesional, el desarrollo de la investigación yla divulgación de la cultura.

Estas actividades representan un solo camino de acción que puede ser aprovechado para salvaguardar la riqueza cultural de un país. En efecto, esta consideración no es peregrina ni innovadora. El trabajo realizado desde el espacio universitario en otros objetos del patrimonio cultural ha producido muy buenos resultados. Podríamos citar al respecto numerosos ejemplos. Sin embargo, no podríamos decidir lo mismo en lo que se refiere al fondo antiguo de las universidades.

Por ejemplo, la Universidad Nacional Autónoma de México (UNAM) cuenta con fondos antiguos de gran importancia, incluso si exceptuamos a aquellos que corresponden a la Biblioteca Nacional. En laUNAM existeformación profesional en bibliotecología y dos espacios de investigación especializada, el Instituto de Investigaciones Bibliográficas (IIB) y el Centro Universitario de Investigaciones Bibliotecológicas (CUIB). A pesar de la existencia de estos espacios institucionales, los fondos antiguos de la UNAM no gozan de un reconocimiento especial ni tampoco representan una preocupación relevante ni para la universidad ni para la disciplina bibliotecológica. Por el contrario, aun cuando han sido objeto de ciertas inquietudes, sabemos muy poco dela composición de estos fondos, de su número y de su riqueza.

Las bibliotecas de la UNAM son las más numerosas y ricas del país y por tanto representan una riqueza bibliográfica incuestionable. Pero pese a que la UNAM cuenta con un sistema bibliotecario y un trabajo que ha marcado el desarrollo de otras bibliotecas en el país, los fondos antiguos no han conseguido aún un estatuto de ciudadanía de acuerdo con su importancia. ${ }^{15}$ Por ejemplo, en 1985 se elaboró un trabajo

15 En octubre del 2000, la Dirección General de Bibliotecas comenzó un proyecto dedicado a el Fondo Antiguo de la Biblioteca Central de la UNAM. Se calcula que este fondo está compuesto por 40,000 volúmenes de 1510 a 1950, que se pretende registrar bajo la norma ISAD (A) y las RCAA2. Sin embargo a la fecha no se ha presentado el modelo de registro bibliográfico que se utilizará ni su justificación. Este aspecto es preocupante debido a la existencia de experiencias anteriores que no han utilizado correctamente las metodologías correspondientes, lo que genera identificación equívoca de materiales y por lo tanto pérdida de evidencia histórica. Cfr. Isabel Chong delaCruz... [ $\notin$ al.] "Nuestras bibliotecas. El proyecto Fondo Antiguo dela Biblioteca Central”, en Biblictea Univesitania Vol. 4, no. 1 (enero-junio 2001) 
sobre los "libros raros" de la UNAM en donde ya se definía una situación poco propicia para estos materiales. En diecisiete años la situación que este trabajo planteaba no havariado considerablemente, a pesar de que se recomendaba que "el bibliotecólogo debe aplicar sus conocimientos teóricos así como prácticos para solucionar los problemas en este tipo de colecciones". ${ }^{16}$

Las consideraciones planteadas en este trabajo referidas a su "catalogación, clasificación, ubicación, uso, cuidado y mantenimiento" 17 siguen presentes a la fecha. Empero, esta inquietud profesional no generó ningún fruto importante. Hasta la fecha laUNAM no incluye este importante tema en la formación especializada de la licenciatura en bibliotecología ni en el posgrado, creado recientemente. Tampoco existe una forma de registro adecuada ni normalizada y mucho menos automatizada. También, por supuesto, la elaboración de catálogos bibliográficos del contenido de los fondos antiguos es muy escasa, pese a ser instrumentos que permiten un mayor conocimiento y difusión de esa riqueza cultural.

Este trabajo de la bibliotecología anteriormente citado como otros de la misma disciplina, no han representado más que una preocupación pasajera y sin fortuna en comparación con inquietudes que provienen de otras disciplinas. Entre éstas habría que citar las del Instituto de Investigaciones Estéticas de la UNAM, en donde desde 1991 se consolidó el SeminariodeEsudioda PatrimmioArtísicoque actualmente sigue aportando importantes consideraciones en un coloquio organizado anualmente. También habría que citar a la Facultad del Hábitat de la Universidad de San Luis Potosí, que haintroducido las preocupaciones sobre bienes patrimoniales en su formación profesional y de posgrado.

Afortunadamente esta preocupación por el patrimonio cultural ha consolidado espacios de formación y de investigación en diversas universidades públicas y privadas. Lamentablemente la preocupación predominante siempre ha estado referida a otros bienes culturales, como los inmuebles o los artísticos, y no a aquellos conservados en los fondos antiguos. Sin embargo, desde hace aproximadamente cinco años esta desafortunada propensión ha cambiado si no radicalmente sí exponencialmente. Empero, los frutos que esta tendencia tiene mostrarán sus resultados a muy largo plazo y será entonces cuando se podrá analizar si logró consolidar sus consideraciones más apremiantes.

En esta nueva tendencia cabe mencionar el importante trabajo que se está realizando en la Benemérita Universidad Autónoma de Puebla (BUAP), en donde diversas dependencias y disciplinas están abriendo campo para trabajar los bienes culturales como un problema patrimonial. En este espacio institucional se está observando un renovado interés que no ha dejado delado alos fondos antiguos. No

16 Juan José Calva G onzález y César Augusto Ramírez Velásquez. "Los libros raros en el Sistema de Bibliotecas de la UNAM", enEnantrodeBiblictecanios dd Sistema deBiblidtecas delaUniveridadNacio nal Autónoma deMéxico(4o: 1985: México, D .F.). México: UNAM. D GB, 1986. p. 413

17 Ibid 
hay que olvidar quela BUAP tiene bajo su adscripción auna de las bibliotecas históricas más relevantes del país: la Biblioteca José María Lafragua. Esta biblioteca ha comenzado a desarrollar numerosas iniciativas para rescatar y difundir su rico fondo antiguo, al que se integran desde la catalogación automatizada basada en el modelo de la ISBD (A),18 hasta el planteamiento de la necesidad de contar con una norma universitaria que considere a este acervo como un bien patrimonial en su conjunto.

D esde este espacio nuevo y por tanto difícil, hemos planteado una investigación para conocer la situación de salvaguarda de los fondos antiguos en las universidades mexicanas. D esde el comienzo de este proyecto, en 1999, hemos observado numerosas dificultades para obtener la información necesaria. Esto nos ha mostrado que la mayor parte de las bibliotecas universitarias que conservan material antiguo, no consideran importante propiciar el conocimiento sobre sus condiciones de salvaguarda. En efecto, el proyecto no ha tenido el eco que hubiésemos deseado; por el contrario, han sido pocas las instituciones que generosamente nos han permitido acercarnos a un universo patrimonial celosamente guardado y prácticamente desconocido.

Este proyecto es una parte derivada de la investigación titulada "El patrimonio D ocumental en México", cuya finalidad es analizar las formas de protección legal que se utilizan para garantizar la salvaguarda de los bienes que integran al patrimonio documental, tal y como se desprende de la definición del programa internacional Memoria del Mundo. Esta investigación, por su parte, pretende analizar las condiciones de salvaguarda de los bienes bibliográficos en el entorno universitario, sea éste público o privado.

Para los fines de esta investigación se entiende por salvaguarda a lo que designa "todas las operaciones, materiales e inmateriales, pero que no conllevan una intervención directa sobre el objeto, destinadas a favorecer la perduración de los bienes culturales en el tiempo". ${ }^{19}$ Así, la investigación se orienta a determinar cuáles son las condiciones de salvaguarda de los fondos antiguos en las bibliotecas universitarias del país. Para obtener la información relacionada con este tema se elaboró un cuestionario ${ }^{20}$ dividido en 10 partes: identificación institucional, tipo de institución, fondos y acervo, organización de los fondos, accesibilidad, preservación y conservación, seguridad, promoción y difusión, financiamiento e infraestructura.

18 En junio de 2002 laBUAP firmó un convenio específico de colaboración con la Universidad de las Américas-Puebla, para realizar conjuntamente un catálogo automatizado de los libros antiguos que ambas instituciones custodian. Este convenio se inserta en un marco de colaboración institucional que formalmente ha reunido por primeravez a estas dos universidades poblanas en 32 años. Este marco institucional representa un esfuerzo compartido nunca antes visto en México para desarrollar proyectos y programas dedicados a la salvaguarda del libro antiguo como un bien patrimonial. Este trabajo representa al mismo tiempo el primer esfuerzo formal en nuestro país para desarrollar un catálogo colectivo de materiales antiguos.

19 Ignacio G onzález Varas. Conservación deBienes Culturales Madrid: Cátedra, 1999. p. 551

20 Este instrumento se elaboró con la colaboración de Luis Villén de la Universidad de G ranada. 
Ahora bien, "el universo de instituciones de educación superior del país, está conformado por 1,259 instituciones", 21 entre las cuales se encuentran tanto las universidades como los institutos tecnológicos. De este conjunto seleccionamos las universidades más representativas por entidad federativa y el número total fue de 52 instituciones, 22 a las cuales se les envío el cuestionario anteriormente citado. También se incluyeron otras cuatro instituciones culturales que poseen un fondo antiguo. D el 2000 al 2002 se enviaron 62 cuestionarios y se obtuvieron respuestas en 36 casos. Así, actualmente contamos con el 58\% de respuestas de la muestra en la primera etapa de la investigación.

Sin duda uno de los mayores problemas de la investigación ha sido la carencia de un instrumento eficaz que nos informe puntualmente sobre la existencia de fondos antiguos en universidades. Son pocas aquellas que cuentan con tales acervos patrimoniales entre sus recursos de información pública einstitucional. Algunos deestos fondos, por tradición y porque sus colecciones son objeto de investigación especializada, son los más reconocidos.

Es preocupante observar que todavía no exista un espacio interinstitucional que al menos compile la existencia de los acervos culturales universitarios, situación contradictoria cuando la difusión de la cultura es un pilar fundamental de la actividad universitaria y considerando que un número importante de universidades poseen bienes culturales muy apreciados. Habría que construir tal espacio en las universidades y ahí mostrarle a la sociedad mexicana este patrimonio para que la población pueda acceder y disfrutar al menos de una parte de su riqueza.

La preocupación por el patrimonio cultural de las universidades no es en absoluto una idea nueva sino una preocupación constante de algunas instituciones, por eso su evolución dentro de la estructura universitaria presenta diversos matices. Existen universidades que poseen direcciones generales especialmente dedicadas a la conservación y custodia de bienes culturales, otras que han creado espacios de formación especializada, aun otras que poseen dependencias como museos universitarios e incluso aquellas en donde estos acervos no tienen ninguna representación.

En este panorama cultural universitario el fondo antiguo delabiblioteca casi pasa desapercibido. Son muchas las razones que podrían explicar esta situación, pero en gran medida los responsables de este olvido somos los bibliotecarios profesionales. Los materiales que componen los fondos antiguos han estado ahí desde que se integraron al espacio universitario y el descuido nuestro ha mermado considerablementelas colecciones. Pero no podemos conocer el límite y la dimensión de esta tragedia cultural en tanto no se considere importante cuando menos su registro más elemental. ¿Cómo saber el número original de materiales que integraron cada uno de estos

21 Tipdoǵa deInsituciones deEduraaón Superior. ANUIES. D atos disponibles en http:/ / www.anuies.mx/ anuies/ libros98/ lib13/ 000.htm [Consultado: Junio 2002]

22 En el caso particular de laUNAM, se envío el cuestionario a las dependencias que se presupone poseen un fondo antiguo relevante. 
acervos? ¿Cómo construir un futuro diferente para estos importantes acervos patrimoniales, tanto para la investigación especializada como para la sociedad en su conjunto, si no conocemos ni siquiera cuál es el monto de esta riqueza? En efecto existe una respuesta co herente: "sólo hay un modo de entrever el futuro: mirando al pasado , pues es profundizando en éste, y desde él, como se construye el futuro" ${ }^{23}$

\section{UN PATRIMONIO DESCONOCIDO, UN PATRIMONIO EN RIESG O : EL MA- TERIAL DEL FONDO ANTIGUO UNIVERSITARIO}

Replantear el futuro de un fondo antiguo requiere cuando menos reconocer e identificar los materiales que lo integran. En efecto, toda condición de valoración de un bien cultural, independientemente dela naturaleza quelo caracteriza, presuponenecesariamente su conocimiento. Este conocimiento ha representado siempre uno de los mayores problemas y constituye la tarea más importante de la conservación y protección delos elementos patrimoniales. Un libro por sí mismo puede aportar bastante información; sin embargo, asociado, relacionado o comparado con otros, ofrece una imagen cercana del desarrollo cultural de las sociedades que nos precedieron.

El siglo XIX marca sin duda el inicio de la sangría que poco a poco ha ido disminuyendo la riqueza que tuvieron las bibliotecas novohispanas. José María Andrade, Agustín Fisher, Francisco Kaska, Herbert Howe Bancrotf, Nicolás León y Henry R. Wagner, entre otros, son nombres relacionados con el coleccionismo y el saqueo de las bibliotecas mexicanas. Pero también están relacionados con laformación de importantes colecciones que hoy son orgullo de diversas instituciones y, especialmente, gracias a la elaboración de instrumentos de investigación como catálogos y bibliografías.

No obstante a pesar de reconocer la importancia de los fondos antiguos como bienes culturales, existe poca investigación relacionada con el número de volúmenes que pudieron haber contenido las bibliotecas novohispanas y, especialmente, sobre el número de materiales de aquellas que pasaron a formar parte de una biblioteca contemporánea. Para nuestra fortuna todavía se conservan inventarios de la época colonial que pueden informarnos sobre el número y tipo de materiales existentes en las bibliotecas de ese periodo histórico.

Algunos de estosinventarios supervivientes proporcionan detalles bibliográficos y constituyen una fuente histórica invaluable que nos permitiría construir un conocimiento aproximado sobre la dimensión y riqueza de los bienes bibliográficos. También existen inventarios realizados durante el periodo independiente que deben ser incluidos en esta recuperación. Pero como ya ha escrito Fernando Benítez: "No se ha intentado siquiera un mínimo inventario de lo que se perdió y está fuera de mi capacidad hacerlo". ${ }^{24}$

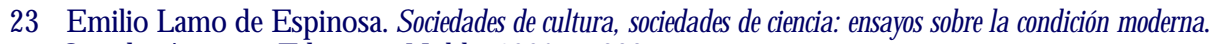
O viedo, Asturias: Ediciones Noble, 1996. p. 220

24 Fernando Benítez. El librodelos desastres México: Era, 1993. p. 72 
Para queun estudio de esta naturaleza sea posible es necesario contar con el registro de los materiales antiguos conservados actualmente en las bibliotecas. Como ya hemos indicado, el registro es el principio fundamental de toda tarea de salvaguarda de los bienes culturales, y en este aspecto a nuestro país le falta mucho por hacer. El registro de los materiales del fondo antiguo representa cuando menos dos problemas concretos. El primero es el conocimiento y comprensión de las características de cada objeto, lo que significa precisar las particularidades que distinguen a un objeto de otro. Hemos anotado ya que en México el uso de conceptos para definir los objetos del fondo antiguo es completamente diverso, así que habría que comenzar por definir un lenguaje especializado de uso común. El modelo bibliográfico para la elaboración de ete tipo de registros es el segundo problema. Sobre este asunto particular hay muchas posiciones y aun cuando existe la norma ISBD (A) para los impresos antiguos, en nuestro país no se ha usado como base para solucionar la elaboración de catálogos tal como se ha realizado en otros países. Partir de un modelo bibliográfico común permite intercambiar información pero también reduce de forma importante los riesgos de identificar erróneamente un impreso antiguo.

La situación actual propicia una inadecuada identificación de estos objetos y por tanto un registro impreciso. Podría ser que un objeto fuera identificado y registrado como otro completamente diferente. ¿Cómo detectar estos en miles de registros ya realizados? La tarea es, en efecto, inmensa, de dimensiones semejantes a la realización de un catálogo de nueva cuenta.

La detección de algunos de estos registros que no corresponden con su original sólo es posible mediante la comprobación directa, y por tanto representa una tarea a largo plazo y de gran envergadura. D urante el desarrollo de la investigación hemos encontrado algunos de estos casos que resultan por demás interesantes, el más llamativo de ellos se refiere a un ejemplar de la "Psalmodia Christiana" de Bernardino de Sahagún, impreso por Pedro O charte en 1583, que había reportado tener desde 1954 la Biblioteca Miguel Lerdo de Tejada de la Secretaría de Hacienda y Crédito Público. Esta obra sumamente importantey valiosa ${ }^{25}$ de los impresos mexicanos del siglo XVI y de la que se conservan pocos ejemplares en el mundo, fue identificada erróneamentey presentada públicamente como laPsalmodiaoriginal, cuando en realidad es algo muy distinto. El ejemplar en cuestión es una construcción artificial26 con características muy particulares.

25 Dela obra de este franciscano notable, fue éstalaúnica que vio impresa. Sahagún padeció enormemente todo lo relacionado con sus versiones al náhuatl de asuntos so bre la Sagrada E scritura debido a que en 1577, el Santo O ficio dela Inquisición prohibió terminantemente este tipo de trabajos. Posteriormente esta "Psalmodia" fue censurada en el siglo XIX y perseguida hasta prácticamente conseguir su extinción. Cfr. Miguel León Portilla. BemardimodeSahagún: pioneodelaantropdoǵa Mé xico: UNAM: El Colegio Nacional, 1999. p.165

26 Sobre este "descubrimiento" estamos desarrollando un trabajo específico. 
En México, este problema del registro de los materiales antiguos presenta cuando menos dos peculiaridades que se deben distinguir. La primera es la ausencia de conocimiento de las diferencias sustanciales que distinguen a un impreso antiguo de uno moderno y que determinan los modelos bibliográficos que utiliza uno y otro. Como el registro específico de un objeto de esta naturaleza tiene por finalidad identificar con claridad y de forma individualizada cada uno de los materiales, es indispensable "conocer los procesos de elaboración del manuscrito y del libro anti guo". ${ }^{27}$ La segunda peculiaridad, como hemos mencionado, se refiere a la carencia de estos conocimientos en la formación profesional necesaria para trabajar con estos materiales. D icha formación es la que determina en gran medida la forma de elaborar los registros.

La identificación de los materiales antiguos mediante registros idóneos intenta definir, más que la existencia de una impresión, los detalles que la caracterizan o diferencian, y aquellos aspectos que deben resaltarse del objeto material que se está describiendo. Estos aspectos determinarán la singularidad de un objeto determinado y posibilitarán su reconocimiento como un bien cultural que requiere condiciones especiales de conservación y protección.

Conocer con precisión cuáles y cuántos son los materiales antiguos de los fondos antiguos de las bibliotecas universitarias representa el primer paso para acercarse a un problema patrimonial. D esde esta perspectiva existe también un paso previo: ¿cuáles son las universidades que poseen este tipo de material? Como hemos indicado esta tarea aún no se ha realizado y el proyecto anteriormente citado sólo representa una mirada discreta que pone de manifiesto la dimensión de un problema que presuponemos es de grandes dimensiones.

Los bibliotecarios detodas las épocas han elaborado repertorios y catálogos de libros, documentos y objetos diversos, gracias a los cuales ha quedado registro de la riqueza bibliográfica del pasado. La existencia de estos instrumentos nos permite conocer el camino histórico de un ejemplar o una colección particular. Sin embargo, poco hemos hecho por compilar un repertorio nacional de las bibliotecas del país que conservan estas riquezas. Saber que un libro antiguo, un incunable o un manuscrito se encuentra en una biblioteca, que es posible consultarlo y saber de antemano las condiciones de conservación del mismo, constituye unainformación extremadamente valiosa no sólo para el investigador especializado sino para las mismas autoridades responsables de su salvaguarda.

Sobre este punto tan importante de la salvaguarda, debemos actuar como se ha hecho ya en otros países. En 1975 por ejemplo, Francia realizó una encuesta para determinar el número de volúmenes antiguos que se conservan en sus bibliotecas. El resultado estadístico de esta encuesta determinó que el material conservado anterior a 1820 era de un millón de volúmenes. Este trabajo nacional, sólo sirvió para determinar el número de volúmenes conservados y no se enfocó a determinar el estado

27 Alberto Montaner Frutos. ProntuariodeBildiogafía Gijón: TREA, 1999. p. 76 
de conservación de los acervos. Efectivamente éste sólo fue el primer paso. Hoy se sabe que solamente en las bibliotecas públicas de Francia, se conservan unos cinco millones de volúmenes antiguos. ${ }^{28}$

D ada la importancia que estas fuentes tienen para la investigación especializada, el interés nacional francés decidió la elaboración de las recomendaciones de 1988 de los Centres d'acquisition \& dediffusion del'informationsientifique $€$ tehmique(CAD IST) ${ }^{29}$ Siendo la conservación de los materiales uno de los puntos focales de la preocupación francesa, los CAD IST obligan a las instituciones a conservar en buenas condiciones el patrimonio documental que custodian. D e esta manera, se impulsa que las instituciones que conservan material antiguo consoliden todas las garantías de seguridad en sus establecimientos, mantengan la unidad de los fondos originales y favorezcan el tratamiento del material por personal cualificado.30

Al igual que en otros países de Europa, el conocimiento de la realidad de los fondos antiguos franceses propició el aumento de los recursos estatales para la conservación, desinfección, microfilmación, digitalización y desacidificación de los materiales conservados en instituciones públicas. En suma, se incrementaron las acciones en favor del patrimonio bibliográfico.

Un año después de la elaboración de estas recomendaciones, la DirectionduLiveet delaLeturedel Ministerio de Cultura Francés, estableció el programanacional denominado "Patrimonio". A través de este esfuerzo se ha conseguido aumentar los recursos financieros de 4 a 14 millones de francos, entre la década de los setentay la de los noventa. Una parte importante del financiamiento recuperado se utiliza para la puesta en valor, la restauración y, de forma importante, para las exposiciones y la promoción de las colecciones de material antiguo.

En este nuevo entorno nacional, las bibliotecas universitarias francesas que cuentan con ricos fondos antiguos están participando activamente, aun cuando dependen de otros polos del patrimonio estatal francés para la investigación, la construcción de instalaciones especializadas e incluso para la formación de restauradores. D entro de este contexto se ha determinado un modelo de relación interinstitucional basado en contratos plurianuales entre el Estado y las universidades para trabajar en la salvaguarda de los fondos antiguos.

El desempeño de Francia es sólo un ejemplo de lo que puede hacerse por la recuperación de fondos antiguos nacionales y universitarios. Los países europeos se han distinguido por la defensa de su patrimonio mediante acciones conjuntas y coordinadas y la elaboración de políticas culturales y de programas específicos. En estepanorama europeo se observa que la atención de los asuntos bibliotecarios trabaja los diferentes problemas de forma integral y no aisladamente. D e esta manera se han

28 Habría que considerar que estos estudios no consideraron ni reconocieron la riqueza de los fondos del siglo XIX.

29 Institución del Estado Francés creada en 1980.

30 Denis Pallier. "La fonction patrimoniale". En Lesbiblidhèquesdansl'Univesitél sous la direction de D aniel Renoult. París: Éditions du Cercle de la Librarie, 1994 p. 163 
obtenido mejores y más eficaces resultados no sólo en la salvaguarda de los fondos antiguos sino en todos los aspectos relacionados.

Estas acciones también han contribuido a mejorar el conocimiento, y por tanto la salvaguarda de estos acervos, eimpulsado la realización de proyectos específicos entre las universidades europeas. La información en materia de cultura se coordina desde un espacio específico dentro de la Comunidad Europea ${ }^{31}$ y bajo acuerdos que han sido negociados por los estados que la integran. También se ha desarrollado un espacio compartido para que colaboren los museos, archivos y bibliotecas en el estudio, la construcción y el diseño de contenidos culturales en los entornos digita les. ${ }^{32}$ La perspectiva comunitaria se orienta desde dos puntos de vista sustanciales: el valor cultural de todos los objetos del patrimonio como signo de identidad europea, y su potencial como factor para el desarrollo económico de la región.

Acogidos por estos espacios interinstitucionales y supranacionales, se crean y consolidan proyectos tan importantes como GABRIEL, 33 que desde 1995 es el espacio en la red que representa a las bibliotecas nacionales europeas. Pero también espacios de trabajo más específicos en donde se encuentran representados materiales de fondos antiguos universitarios, como el Consorcio de Bibliotecas de Investigación de Europa ${ }^{34} 0$ el G rupo de Bibliotecas de Investigación del Reino Unido. ${ }^{35} \mathrm{Un}$ caso semejante ocurre en los Estados Unidos, donde sus ricos fondos antiguos universitario han cobrado la importancia y el reconocimiento que merecen en las políticas nacionales y en proyectos específicos.

Pero también en América Latina los fondos antiguos están cobrando un renovado interés en países como Argentina, Brasil, Perú o Chile. Ciertamente las posibilidades tecnológicas empleadas para rescatar y difundir estos acervos culturales en Europa y los Estados Unidos rebasan, con mucho, el trabajo latinoamericano. Lo que realmente resulta extraño es que frente a la riqueza conservada en varias de las bibliotecas del país, estemos ausentes en la red con proyectos tecnológicos que permitan conocer y difundir el legado cultural que representan estos materiales.

Al igual que en otros países, "en el campo nacional, la característica más notable de los fondos antiguos universitarios, es la presencia al mismo tiempo de grandes colecciones enciclopédicas y especializadas de primer nivel" ${ }^{36}$ La pregunta es ¿por qué no estamos actuando en la conservación y rescate de esta riqueza? ¿Por qué no

31 European Culture Portal. Información disponible en http:/ / europa.eu.int/ comm/ culture/ index_en.htm [Consultado: junio 2002]

32 Digicult. Información disponible en http:/ / www.cordis.lu/ ist/ ka3/ digicult/ home.html [Consultado: junio 2002]

33 Gabriel. The gateway to Europe's National Libraries. Información disponible en http:/ / www.bl.uk/ gabriel [Consultado: mayo 2002]

34 Consortium of European Research Libraries. Información disponible en http:/ / www.cerl.org/ [Consultado: junio 2002]

35 Research Libraries Group. Información disponible en http:/ / www.rlg.org/ [Consultado: junio 2002]

36 Denis Pallier. Op àt. p. 161-62 
estamos invirtiendo en desarrollar proyectos que le permitan ala sociedad mexicana y a la comunidad internacional acceder a este universo patrimonial tan exquisito? Prácticamente ninguno de los recursos disponibles actualmente en la red sobre fondos antiguos incluye trabajos o proyectos realizados por instituciones de educación superior de México.

Uno de los grandes problemas que representa la salvaguarda del patrimonio es garantizar el acceso y el disfrute de los bienes patrimoniales. Sin registro, sin recursos, sin conocimiento, los fondos antiguos en México enfrentan una difícil situación. Integrar en un solo espacio institucional la formación profesional y el desarrollo de la investigación a la vez que difundir en la sociedad -a través de sus servicios culturales- los resultados obtenidos en el rescate de los fondos antiguos no es una tarea imposible para las universidades. Éstas son un espacio capaz de coordinar los esfuerzos y mejorar la situación de los fondos antiguos del país y devolverles así la naturaleza cultural que los caracteriza. Es decir, un legado que se transmite entre generaciones. El reto es importante, pero no se podrán realizar acciones concretas si no actuamos como un grupo orientado hacia un único fin: la salvaguarda de la memoria documental del país.

\section{LAS CONDICIONESDE LA SALVAGUARDA DEL FONDO ANTIGUO UNI- VERSITARIO: UN GRAVE PROBLEMA QUE DEBEMOSENFRENTAR}

Como hemos argumentado en líneas anteriores una de las primeras tareas es identificar la existencia de fondos antiguos en México. El proyecto "Conocer nuestra memoria documental", quellevamos a cabo en el CUIB, pretende en primer lugar identificar los fondos antiguos de las universidades en México tanto públicas como privadas. Nos interesa especialmente conocer las condiciones generales de salvaguarda que existen en las instituciones que custodian un fondo antiguo.

Por salvaguardar, como hemos ya explicado, se entiende "cualquier medida de conservación que no implique la intervención directa sobre la obra". ${ }^{37}$ Significa, por tanto, garantizar todas las acciones y medios necesarios para conservar íntegramente un objeto cultural de la mejor manera posible. Frente a esta idea se contrapone una realidad que determina la vulnerabilidad y fragilidad de los materiales que se conservan en los fondos antiguos de las bibliotecas: no existe un material más sensible a los cambios de temperatura, humedad y luz, que estos viejos testimonios.

Sin embargo, la condición de conservación que narra el devenir histórico de cada material, nos muestra que dicha fragilidad a veces está aco mpañada de una verdadera fortaleza. Pero esta conservación tan extraordinaria de algunos materiales no se debe ciertamente a una constante preocupación institucional y social. En la mayor

37 “Carta del Restauro de 1972". En la obra deCesare Brandi. Teeńadelarestauracón Madrid: Alianza. 1999. p. 131. 
parte de los casos han sido las azarosas circunstancias las que han permitido que unos materiales sean verdaderos supervivientes frente a otros. Empero, existen aquellos que languidecen ya tan sólo para recordarnos el límite de la tragedia: la pérdida irreparable de un testimonio histórico.

"En los libros se encuentra guardado lo mejor del hombre: su historia, su pensamiento, sus vivencias, su imaginación, su humor, etc.; pero los enemigos que acechan a estos débiles seres son numerosos y muy destructivos" ${ }^{38}$ Entre estos habría que considerar como agente importante de la destrucción a la acción del hombre. En efecto, la conservación de los materiales del fondo antiguo de la biblioteca se ve afectada tanto por los agentes biológicos y químicos, como por el desgaste producido por el uso y también por la negligencia en su conservación.

En la investigación que actualmente estamos desarrollando sobre los fondos antiguos de las universidades se preguntó a las instituciones en un apartado especial del cuestionario, sobre el estado de conservación de los fondos de la institución; las respuestas obtenidas son sumamente interesantes. Nuestra investigación cuenta actualmente con 36 respuestas institucionales al cuestionario enviado, de las cuales cuatro no reportan la existencia de fondos de esta naturaleza. En términos generales nos interesa conocer si las instituciones tienen salas especialmente acondicionadas para albergar estos fondos, si cuentan con ambientes de temperatura y humedad controlada, y cuál es, en su opinión, el estado general de la conservación de sus materiales.

De los datos resultantes de este primer acercamiento y muestreo a las instituciones, un porcentaje del $63 \%$ deéstas consideran que su fondo se encuentra en buenas condiciones; el 22\% considera que su estado de conservación es regular; el 9\% considera que es muy bueno y sólo el $6 \%$ reporta que la conservación es mala. Por otra parte, el 75\% de la muestra nos informa que cuentan con salas especiales para albergar estos fondos. Respecto a las condiciones de estos espacios sólo el 50\% nos informa que existen mecanismos para controlar la temperatura y el $60 \%$ para controlar la humedad.

Como puede observarse en estos datos la información no coincide, si la mayor parte cuenta con salas especiales ¿por qué no coinciden las medidas básicas de conservación como la temperatura y humedad controlada? Por otro lado al preguntársele a las instituciones acerca de actuaciones directas sobre los materiales conservados se reportó que sólo el 31\% cuenta con laboratorio o taller de restauración; el $62 \%$ reporta daños importantes del papel en los materiales; el 50\% realiza reparaciones internas de los materiales; el 67\% realiza tareas de encuadernación cuando los materiales lo requieren y, finalmente, en el $46 \%$ de las instituciones el material es limpiado por personal especializado. Si contrastamos el 63\% de la muestra que considera que su conservación es buena, con el 62\% que reporta daños importantes en el papel, volvemos a encontrar que los datos no coinciden.

38 Pablo Antón Melero. Introduccoónalarestauracónartesanal delihros, grabadbsymanuscritos Madrid: O llero \& Ramos, 1995. p. 25 
Lamentablemente esta misma particularidad se observa respecto al registro e inventario de los materiales, tarea que es crucial para el establecimiento de políticas y programas específicos de salvaguarda de los fondos antiguos. Respecto a este punto, el cuestionario solicitó información a las instituciones sobre la existencia de inventario acerca de los materiales, los instrumentos de descripción y las formas de control automatizado. También se preguntó so bre la cifra total del acervo, el número de ejemplares por siglo, los materiales anteriores al siglo XVI como los incunables, la procedencia de los fondos y la existencia de documentación probatoria de esta procedencia.

Las respuestas proporcionadas sobre estas preguntas no son claras ni específicas e incluso podríamos calificarlas de inconsistentes. Aun con esta característica, la muestra nos estaría permitiendo deducir que al menos se conservan aproximadamente en estos fondos antiguos un millón y medio de volúmenes incluidos los del siglo XIX.

Se trata de una muestra muy pequeña de la riqueza que suponemos existe. El cuestionario apuntala nuestra hipótesis sobre la diversidad conceptual relacionada con el fondo antiguo y, más importante aún, sobre la carencia nacional de un registro e inventario de los objetos culturales de esta naturaleza que se conserva ¿Cómo conservar y proteger un conjunto de objetos que no se conoce plenamente? ¿Cómo saber cuáles de estos objetos son únicos en el mundo? ¿Cómo determinar cuáles objetos son los más relevantes del acervo patrimonial?Y finalmente ¿cómo construir una política nacional de salvaguarda de estos objetos si ni siquiera sabemos dónde están?

En los trabajos internacionales sobre las rondas antiguas se observa que el comienzo delas preocupaciones patrimoniales se inició con la realización de encuestas que han permitido determinar el número de ejemplares antiguos existentes en las bibliotecas universitarias. Sin embargo en México a esta tarea se suma una todavía más importante: identificar cuáles instituciones tienen este tipo de materiales. Posteriormente se requeriría cubrir también una de nuestras lagunas mas abismales: inventariar y registrar las colecciones patrimoniales.

Registrar e inventariar son tareas que deben realizarse de forma paralela. Sin embargo, como hemos planteado, el registro de este material no resulta tan sencillo. Sin conocimiento delas características sustanciales se pueden cometer errores de extrema gravedad. La catalogación del material antiguo actualmente se realiza bajo el modelo de las ISBD (A) y dicha norma internacional es el resultado de un trabajo exhaustivo, pero en nuestro país esta norma no se enseña en la formación profesional. Considerando que para comprender y utilizar esta norma paralelamente se deben enseñar las peculiaridades del material antiguo, y sabiendo que este conocimiento no tiene espacio en laformación bibliotecológica, tendremos que reconocer que el problema del registro de estos bienes adquiere una dimensión todavía más compleja.

Entendemos por catalo gación a la "representación normalizada de los datos documentales para la localización física de los documentos ordenados en catálogos según 
criterios determinados" ${ }^{39}$ En la bibliotecología la enseñanza de este proceso hace hincapié en que la identificación de un material tiene dos fuentes primarias de información: la portada y el colofón. Por el tipo de casos de identificación errónea de un material antiguo que hemos encontrado, esta idea resulta ser uno de los problemas principales.

Si la persona que realiza un catalogo de material antiguo sólo se queda con esta mirada sobre el objeto posiblemente cometerá errores. Una de las características que distingue deforma regular alos incunables, manuscritos y librosantiguos conservados en losfondos antiguos es la existencia de elementos históricos. Elementos que tienen dos caras: la propia de los detalles de impresión y, aquella que es el resultado del devenir histórico de un objeto. Entre estos últimos podemos distinguir las marcas de fuego, los ex libris, las anotaciones manuscritas y, por supuesto, la encuadernación.

Considerando esta evidencia, si nos fijamos específicamente en la encuadernación y más aún si es de época, observaremos que una gran parte de ellas tienen hojas impresas al principio y al final del material, que pueden ser completamente distintas a la obra en cuestión. Éstas son las denominadas hojas guardas, que en ocasiones pueden ser parte de otro material y conducir a error si no se verifica el original completo. Ciertamente entre estas hojas hay algunas que son evidentemente guardas, pero eso no elimina la posibilidad de que otras sean algo muy diferente y puedan inducir al error.

En otros casos, al registrar la información de portada de un material para elaborar el registro bibliográfico correspondiente y no encontrar datos de impresión se acude, por norma general, a la información del colofón. Sin embargo, en aquellos casos en que no se observó que el material es un volumen facticio (es decir, que la encuadernación comprende más de una obra), se registran los datos de autor y el título del primer texto existente, y los datos de impresión del último. Estos casos de registro han creado materiales inexistentes, porque nunca fueron impresos de la forma en que fueron luego registrados.

Aquí existe otro punto importante en relación con estos volúmenes facticios; cuando se registran, acertada o erróneamente, de forma general sólo se refieren a una obra (la primera) y no a todas las que integran dicho volumen. Si trasladamos este registro al inventario de los materiales, observaremos claramente que los datos nunca corresponderán con la realidad del acervo. En efecto el número de volúmenes no puede ser igual al número de obras conservadas. En esta relación yace la importancia que tiene el registro con el inventario de materiales antiguos.

Lasituación prevaleciente en laformación profesional del bibliotecólogo no ayuda en nada a favorecer el conocimiento sobre los fondos antiguos en México. Ciertamente ésta es sólo una parte del inmenso problema que como bienes culturales

39 José Martínez de Sousa. Diciananiodebiblidoǵayaienasafaines Madrid: Fundación G ermán Sánchez Ruipérez, 1993. p. 135 
representan. Pero al no existir espacio en la formación profesional para estos te mas, ${ }^{40}$ es muy poco probable que se logren consolidar líneas y temas de investigación especializados, como ocurre en otros países. D esde esta perspectiva el trabajo universitario resulta crucial, especialmente sopesando que la adecuada salvaguarda de los fondos antiguos requiere, como otros aspectos del patrimonio cultural, de un trabajo multidisciplinario.

Las comparaciones son odiosas, pero a diferencia de otros países en donde la función patrimonial está presente en las grandes bibliotecas de enseñanza superior, en México la cuestión de un patrimonio que representa una herencia cultural parece estar completamente abandonada. Estos fondos representan la posibilidad de proporcionarle una documentación coherente a cualquier investigación histórica, pero los problemas de conservación, organización y facilidad de acceso han mostrado que la solución sólo es posible con acciones cooperativas. ${ }^{41}$

"En un país como México la historia de las bibliotecas no necesita justificación ya que cuenta con una de las tradiciones culturales más antiguas de América” . ${ }^{2}$ Pero esta opinión no toma en cuenta la importancia de historiar también los acervos y los materiales que éstos contienen. La biblioteca que posee este tipo de acervo debe trabajar no sólo por registrar e inventariar su riqueza, sino también por conocer sus materiales para identificar los más valiosos. La función social de un acervo de esta naturaleza es prioritariamentela conservación y, ésta debe incluir la noción de quela rareza y la fragilidad limitarán necesariamente el acceso a tales o bras. Por ello la institución debe también reflexionar sobre las mejores maneras de propiciar el conocimiento social de estos bienes a través de su difusión yuso, sin poner en riesgo laintegridad de los originales.

Frente a lo mucho que se ha escrito sobre los objetos que conforman el patrimonio que integra los fondos antiguos universitarios, es importante disertar sobre lo que determina esta composición en términos de relación entre objetos y el devenir histórico que los agrupo en un solo lugar. Un problema también por dilucidar, son los rasgos comunes de una realidad social e institucional "nacional" que tome en cuenta la diversidad y la originalidad de cada universidad. 43

La conservación del patrimonio conservado en las bibliotecas universitarias es para algunos individuos un deber y para otros un placer. Pero también persiste la idea de que este patrimonio y su riqueza es un secreto que no debe ser revelado. Considerar y comprender que los bienes de estos fondos representan una riqueza

40 Habría que indicar que la existencia de cursos y diplomados que se han impartido sobre estos temas, no logra constituir un programa de formación específico.

41 Dennis Pallier. Op at. p. 160

42 "Presentación", en Carmen Vázquez Mantecón, Alfonso Flamenco Ramírez y Carlos Herrero Bervera. Las bibidecas mexicanas en e sigoXIX. México: SEP. Dirección General de Bibliotecas, 1987. p. 9.

43 D aniel Renoult. "Avant-propos", en Lesbiblidhèques dansl'Univesité.. Op at p. 8. 
social, y que debe ser dado a conocer el magnifico patrimonio que ahí se custodia es una de las acciones más importantes que esperan su realización.

La tarea que se plantea está muy lejos de ser fácil. Representa una absoluta cruzada que requiere constantemente de ejércitos de soldados convencidos de su tarea. Los materiales del fondo antiguo han esperado pacientemente su turno para acceder al paraíso del patrimonio cultural mexicano ¿No es hora ya de que formen parte de nuestras preocupaciones sociales y culturales? Sólo me queda esperar y confiar que así sea.

\section{CONCLUSIONES}

Indudablemente, los fondos antiguos de las bibliotecas mexicanas representan un universo inimaginable de conocimiento histórico y de posibilidades de estudio que apenas han sido tocados. Sin embargo, ninguna de las acciones realizadas en el pasado han logrado convencernos como sociedad, de la importancia que tienela salvaguarda, en las mejores condiciones posibles, de esta riqueza cultural. Recuperar esas acciones del pasado y darles continuidad es indispensable y representa una oportunidad de futuro.

El panorama actual ofrece muchas oportunidades que no deben ser desaprovechadas, especialmente por las universidades. La causa del libro y de la biblioteca es generalmente de interés público, sin embargo las bibliotecas patrimoniales han sido durante mucho tiempo ignoradas, excepto por los especialistas. Para que ésta se convierta en una causa social debe darse a conocer no sólo su riqueza sino también su problemática. La intención debe guiarse por lo que otros ya han conseguido: despertar en un público más amplio el gusto por su patrimonio escrito. Un aprecio que debe ser transmitido de la misma manera que se ha ido adquiriendo el gusto social por el patrimonio histórico y artístico. Esto sólo es posible si se amplia el acceso del público a los recursos patrimoniales, antes de lo cual es necesario describir y conocer para luego hacer una puesta en valor de esos recursos. Sin embargo, las acciones deben orientarse hacia la construcción de espacios de cooperación e intercambio entre las instituciones y los especialistas. D e esta apuesta dependen los resultados a corto y largo plazo.

Empero el conocimiento público no debe poner en riesgo los originales. Por esta razón nuestro proyecto de investigación también ha buscado compilar un acervo fotográfico que permita una primera mirada a la riqueza, variedad y belleza de las colecciones. Estas imágenes permiten mostrar la diversidad y complejidad de elementos patrimoniales desconocidos por la sociedad mexicana, pero también nos muestran las caras del desastre. A través de ellas podemos acceder y disfrutar de un bien cultural, pero debemos también coadyuvar a crear conciencia sobre su importancia cultural entre el público en general. Consolidar esteinterés es la garantía más idónea 
para transmitirle la preocupación a otras generaciones. Sólo éstas decidirán la continuidad de nuestras acciones.

Sin duda son varias las líneas de acción. De ahí que consideremos importante identificar, en la medida de lo posible, tanto a las instituciones que custodian fondos antiguos como a los objetos mismos. Lamentablemente, frente a la complejidad del problema debemos reconocer que un proyecto de investigación no es suficiente para mejorar las condiciones de salvaguarda ni para estimular el desarrollo de conocimiento especializado. Éstees sólo un esfuerzo del que estamos plenamenteconvencidos.

Precisar los detalles requeriría de un estudio de campo directo sobre las condiciones de salvaguarda pero realizado en el marco de un programa nacional que previamente haya definido sus metas y objetivos con claridad. El trabajo hasta ahora realizado es sólo un primer acercamiento y no puede pretenderse más. No obstante, puede constituir un espacio de reflexión compartida, a eso es a lo que sí le apostamos.

Todas las bibliotecas patrimoniales, grandes y pequeñas, públicas y privadas, estatales y universitarias, antiguas o recientes, están obligadas a perseguir este objetivo: asegurar la salvaguarda de un bien social y trabajar en una defensa común. Pero estas instituciones no pueden conseguir esta tarea por sí solas y mucho menos aisladamente. Unas y otras deben unir sus esfuerzos para seguir existiendo como lugares donde se conservan libros, donde se leen esos libros, y donde se puede encontrar todavía ese producto raro que es siempre el tesoro de toda biblioteca: el silencio que impone el pasado. 44

Si lafunción patrimonial no le interesa alas universidades en proporción directa a su riqueza documental, entonces se impone una pregunta fundamental: ¿a quién le incumbe en México la carga y responsabilidad de conservar las colecciones históricas y especializadas de primer rango para los investigadores del presente y del futuro? Las respuestas a todas nuestras preguntas sólo pueden ser compartidas, de otro modo padecerán de lo mismo que otras han mostrado ya: que todo esfuerzo aislado no tiene ningún futuro. Por eso la tarea más importante es la consolidación de grupos de trabajo e interés orientados a salvaguardar el patrimonio documental.

En efecto, compartirinformación, recursos e intereses parece ser la mejor forma de obtener resultados concretos. Los espacios institucionales ya existen, la opción es convencer a las instituciones sobre la viabilidad de trabajar en éstos aprovechando su experiencia previa, y de este modo ofrecerle a estos testimonios un futuro diferente: la posibilidad de transmitirse en el tiempo.

44 Michel Pastoureau. "Prefacé", en PatriminedeskibidhèquesdeFrance ungiidedesrégions París: Banques CIC pour le Livre: Ministère de la Culture: Payot, 1995. Vol. 1: Ile-de-France. p. 15. 


\section{BIBLIOG RAFÍA CON SU LTADA}

ABID, A bdelaziz. Memriadd Munda conservandonuestropatrimmiodo ammall. México: Comisión Nacional de los Estados Unidos Mexicanos para la UNESCO : UNAM: UAEM: UAEH, 1998

ALCÁNTARA HEWITT, Rebeca. Unanálisiscúticodelateenáa delares tauradóndeCesareBrandi. México: INAH, 2000.

ANTÓN MELERO, Pablo. Introducióna la restauracón artesanal delibros, gabadosymanuscritos Madrid: O llero \& Ramos, 1995.

Lesbiblidhèquescansl'Univesitél sous la direction de D aniel Renoult. París: Éditions du Cercle de la Librarie, (C1994.

BENÍTEZ, Femando. El librodelos desastres México: Era, 1993.

BRANDI, Cesare. Tẹna dela restauradón Madrid: Alianza, 1999.

BUSTAMENTE ROD RÍGUEZ, Antonio Tomás. "20 años de política bibliotecaria en Andalucía". p. 49-67, en BdéíndelaAsociacoón Andaluza deBiblictecarios No. 63 (junio 2001)

CALVA GONZÁLEZ, Juan José y César Augusto Ramírez Velásquez. "Los libros raros en el Sistema de Bibliotecas de la UNAM". p. 411-432. En Enarentro deBiblictearios di Sistema de Biblidteas dela Univesidad Naional A utónoma deMéxico (40: 1985: México, D.F.) México: UNAM. D GB, 1986.

CAMPILLO GARRIGOS, Rosa. La gestión y e getor de patrimonio altural. Murcia: ediciones KR, 1998.

CHECA Cremades, José Luis. El libroantiguo Madrid: Acento Editorial, 1999.

GARCÍA, Idalia y Miguel Ángel Rendón. "El fondo antiguo: su estructura conceptual". En Binaria: reista decommicacoón alturay temdoǵa Vol. 1 (diciembre 2000).

D isponible en http:/ / www.uem.es/ binaria/

GASKELL, Phillip. Nuea introdurcoón a la bibliogafía mateial. G ijón: TREA, 1999.

GONZÁLEZ VARAS, Ignacio. Conservacóndebienesailturales Madrid: Cátedra, 1999.

HAEBLER, Konrad. Introdución al estudio de los imamables Madrid: Ollero \& Ramos, 1995

HERRERA MORILLAS, José Luis. "el fondo antiguo de las bibliotecas universitarias de Andalucía, Extremadura y Murcia: colecciones, textos normativos y recursos virtuales". p. 55-73, en Bdefín Andaluza deBildidteanios No. 64 (septiembre 2001)

Ex librisunivesitatis e patrimoniodelasbiblictecasunivesitariasespañdas Madrid: Conferencia de Rectores de las Universidades Españolas, 2000. 
LEÓN PORTILLA, Miguel. Bemardino de Sahagún: pionero de la antropoloǵa México: UNAM: El Colegio Nacional, 1999.

MARSÁ VILA, Maria. El fondb antiguo en la biblidtea Gijón: TREA, 1999.

MARTÍNEZ DE SO USA, José. Dicionariodebibidoǵayäenaasafines Madrid: Fundación G ermán Sánchez Ruipérez, 1993.

MELERO, Pablo Antón. Introdurciónalarestauradónartesanal delibros, gabadosymanuscritos Madrid: Ollero \& Ramos, 1995.

MENEGUS BORNEMANN, Margarita y Armando Pavón Romero. "La Real y Pontificia Universidad de México. Panorama historiográfico". p. 67-80. En Historia dela Univeridad Cdonial: avanes deinvestigaaión México: UNAM. CESU, 1987.

MORALEJE ÁlVAREZ, María Remedios. “El patrimonio bibliográfico en las universidades españolas". p. 227-259, en Bdeín dela ANABAD. Vol. 49, no. 2 (1998)

MONTANER FRUTOS, Alberto. Prontuariodebibliogafía: pautas para la realizacón dedsscipiones, atasyrepatarios Gijón: TREA, 1999.

MORALEJO ÁLVAREZ, M. R. "El patrimonio bibliográfico de las universidades españolas". p. 227-259, en Bdéíndela ANABAD, XLIX (1998)

O SORIO ROMERO, Ignacio. Las biblicteras novohispanas México: SEP. Dirección G eneral de Bibliotecas, 1986.

Patriminedesbiblidhèques deFrance unguidedes régions París: Banques CIC pour le Livre: Ministère de la Culture: Payot, 1995. Vol. 1: Ile-de-France

SALAZAR IBARG ÜEN, Columba. Unabibidtee vimenal dePudda, sigoXV III: FondoAndrésdeArzeyMiranda Puebla: Benemérita Universidad Autónoma de Puebla. Instituto de Ciencias Sociales y Humanidades, 2001

VÁZQUEZ MANTECÓN, Carmen, Alfonso Flamenco Ramírez y Carlos Herrera Bervera. Lasbiblicterasmexicanasend sigoXIX. Mé xico: SEP. D irección G eneral de Bibliotecas, 1987.

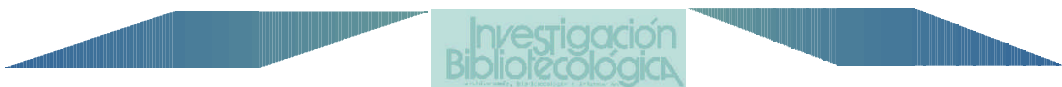

